\title{
Equilibrium Fermi-liquid coefficients for the fully screened $N$-channel Kondo model
}

\author{
M. Hanl, ${ }^{1}$ A. Weichselbaum, ${ }^{1}$ J. von Delft, ${ }^{1}$ and M. Kiselev ${ }^{2}$ \\ ${ }^{1}$ Physics Department, Arnold Sommerfeld Center for Theoretical Physics and Center for NanoScience, \\ Ludwig-Maximilians-Universität München, 80333 München, Germany \\ ${ }^{2}$ The Abdus Salam International Centre for Theoretical Physics, Strada Costiera 11, I-34151 Trieste, Italy
}

(Dated: May 27, 2014)

\begin{abstract}
We analytically and numerically compute three equilibrium Fermi-liquid coefficients of the fully screened $N$-channel Kondo model, namely $c_{B}, c_{T}$ and $c_{\varepsilon}$, characterizing the magnetic field and temperature-dependence of the resisitivity, and the curvature of the equilibrium Kondo resonance, respectively. We present a compact, unified derivation of the $N$-dependence of these coefficients, combining elements from various previous treatments of this model. We numerically compute these coefficients using the numerical renormalization group, with non-Abelian symmetries implemented explicitly, finding agreement with Fermi-liquid predictions on the order of $5 \%$ or better.
\end{abstract}

PACS numbers: 05.10.Cc, 71.10.Ay, 73.63.Kv, 72.15.Qm

\section{INTRODUCTION}

The Kondo effect was first observed, in the 1930s, for iron impurities in gold and silver ${ }^{1,2}$, as an anomalous rise in the resistivity with decreasing temperature. Kondo ${ }^{3}$ showed that this effect is caused by an antiferromagnetic exchange coupling between the localized magnetic impurity spins and the spins of the delocalized conduction electrons $^{3}$, and based his arguments on a spin- $\frac{1}{2}$, oneband model. While this model undoubtedly captures the essential physics correctly in a qualitative way, it has recently been shown ${ }^{4,5}$ that a quantitatively correct description of the Kondo physics of dilute Fe impurities in $\mathrm{Au}$ or Ag requires a fully screened Kondo model involving three channels and a spin- $\frac{3}{2}$ impurity. This conclusion was based on a comparison of temperature and magnetic field dependent transport measurements ${ }^{4-6}$ to theoretical predictions for fully screened Kondo models with channel number $N$ and local spin $S$ related by $N=2 S$, with $N=3$ yielding much better agreement than $N=1$ or 2 .

The theoretical results in Ref. 5 were obtained using the numerical renormalization group (NRG), ${ }^{7-10}$ and for $N=3$ various non-Abelian symmetries ${ }^{5,11}$, such as $\mathrm{SU}(2) \times \mathrm{U}(1) \times \mathrm{SU}(N)$, had to be exploited to achieve reliable results at finite magnetic field. The technology for implementing non-Abelian symmetries with $N>2$ in NRG calculations has been developed only recently. ${ }^{11,12}$ Given the complexity of such calculations, it is desirable to benchmark their quality by comparing their predictions to exact results. The motivation for the present paper was to perform such a comparison for the low-energy Fermi-liquid behavior of fully screened Kondo models, as elaborated upon below.

All fully screened Kondo models feature a ground state in which the impurity spin is screened by the conduction electrons into a spin singlet. The low-energy behavior of these models can be described by a phenomenological Fermi-liquid theory (FLT) formulated in terms of the phase shift experienced by conduction electrons that scatter elastically off the screened singlet. Such a description was first devised for the simplest case of $N=1$ by Nozières ${ }^{13,14}$ in 1974, and generalized to the case of arbitrary $N$ by Nozières and Blandin $(\mathrm{NB})^{15}$ in 1980 . Their results were confirmed and elaborated by various authors and methods, including NRG, ${ }^{7,8,16-20}$ field-theoretic calculations, ${ }^{21,22}$ the Bethe Ansatz, ${ }^{23,24}$ conformal field theory (CFT), ${ }^{25,26}$ renormalized perturbation theory, ${ }^{27}$ and reformulations ${ }^{28-30}$ and generalizations ${ }^{31-33}$ of Nozières' approach in the context of Kondo quantum dots.

In the present paper, we focus on three particular Fermi-liquid coefficients, $c_{B}, c_{T}$ and $c_{\varepsilon}$, characterizing the leading dependence of the resistivity on magnetic field $(B)$ and temperature $(T)$, and the curvature of the equilibrium Kondo resonance as function of excitation energy $(\varepsilon)$, respectively. Explicit formulas for all three of these coefficients are available in the literature for $N=1$, but for general $N$ only for the case of $c_{T}$. Given the wealth of previous studies of fully-screened Kondo models, the lack of corresponding formulas for $c_{B}$ and $c_{\varepsilon}$ was somewhat unexpected. Thus, we offer here a unified derivation of all three Fermi-liquid coefficients, $c_{T}, c_{B}$ and $c_{\varepsilon}$. We follow the strategy which Affleck and Ludwig (AL) ${ }^{26}$ have used to reproduce Nozières' results ${ }^{13}$ for $N=1$, namely doing perturbation theory in the leading irrelevant operator, and generalize it to the case of arbitrary $N$. Our formulation of this strategy follows that used by Pustilnik and Glazman $(\mathrm{PG})^{29}$ for their discussion of Kondo quantum dots. While all pertinent ideas used here can be found in the literature, we hope that our rather compact way of combining them will be found useful.

For our numerical work, we faced two challenges: First, the complexity of the numerical calculations increases rapidly with increasing $N$; this was dealt with by exploiting non-Abelian symmetries. Second, numerical calculations do not achieve the scaling limit that is implicitely presumed in analytical calculations; its absence was compensated by using suitable definitions of the Kondo temperature, following Ref. 34 .

The paper is organized as follows. In Sec. II we define the model and summarize our key results for the Fermiliquid coefficients $c_{B}, c_{T}$ and $c_{\varepsilon}$. Section III compactly 
summarizes relevant elements of FLT and uses them to calculate these coefficients. Section IV describes our numerical work and results. Section V summarizes our conclusions.

\section{MODEL AND MAIN RESULTS}

The fully-screened Kondo model for $N$ conduction bands coupled to a single magnetic impurity at the origin is defined by the Hamiltonian $H=H_{0}+H_{\text {loc }}$, with

$$
\begin{aligned}
H_{0} & =\sum_{k m \sigma} \xi_{k} c_{k m \sigma}^{\dagger} c_{k m \sigma}, \\
H_{\mathrm{loc}} & =J_{\mathrm{K}} \sum_{k k^{\prime} m \sigma \sigma^{\prime}} c_{k m \sigma}^{\dagger} \frac{\vec{\tau}_{\sigma \sigma^{\prime}}}{2} c_{k^{\prime} m \sigma^{\prime}} \vec{S}-B S_{z} .
\end{aligned}
$$

Here $H_{0}$ describes $N$ channels of free conduction electrons, with spin index $\sigma=(+,-)=(\uparrow, \downarrow)$ and channel index $m=1, \ldots, N$. We take the dispersion $\xi_{k}=\varepsilon_{k}-\varepsilon_{\mathrm{F}}$ to be linear and symmetric around the Fermi energy, $\xi_{k}=k \hbar v_{\mathrm{F}}$. Each channel has exchange coupling $J_{\mathrm{K}}$ to a local SU(2) spin of size $S=N / 2$ with spin operators $\vec{S}$, and $B$ describes a local Zeeman field in the $z$-direction (we use units $g \mu_{B}=1$ ). The overall symmetry of the model $^{19}$ is $\mathrm{SU}(2) \times \mathrm{Sp}(2 N)$ for $B=0$, and $\mathrm{U}(1) \times \operatorname{Sp}(2 N)$ for $B \neq 0$ (see Sec. IV A for details). The model is characterized by a low-energy scale, the Kondo temperature, $T_{\mathrm{K}} \sim \tilde{D} \exp \left[-1 /\left(\nu J_{\mathrm{K}}\right)\right]$, where $\nu$ is the density of states per channel and spin species and $\tilde{D}$ is of the order of the conduction electron bandwidth.

For a disordered metal containing a dilute concentration of magnetic impurities, the magnetic-impurity contribution to the resisitivity has the form ${ }^{5,35}$

$$
\rho(T, B) \propto \int d \varepsilon\left(-\partial_{\varepsilon} f(\varepsilon, T)\right) \sum_{m \sigma} A_{m \sigma}(\varepsilon, T, B) .
$$

Here $f(\varepsilon, T)$ is the Fermi function, and the impurity spectral function $A_{m \sigma}(\varepsilon)=-\frac{1}{\pi} \operatorname{Im} \mathcal{T}_{m \sigma}(\varepsilon)$ is the imaginary part of the $T$ matrix $\mathcal{T}_{m \sigma}(\varepsilon)$ describing scattering off a magnetic impurity. The latter is defined through ${ }^{37,38}$

$$
\begin{aligned}
\mathcal{G}_{m \sigma, \mathbf{k}, \mathbf{k}^{\prime}}^{c}(\varepsilon) & =\mathcal{G}_{m \sigma, \mathbf{k}}^{0}(\varepsilon) \delta\left(\mathbf{k}-\mathbf{k}^{\prime}\right) \\
& +\mathcal{G}_{m \sigma, \mathbf{k}}^{0}(\varepsilon) \mathcal{T}_{m \sigma}(\varepsilon) \mathcal{G}_{m \sigma, \mathbf{k}^{\prime}}^{0}(\varepsilon),
\end{aligned}
$$

with $\mathcal{G}_{m \sigma, \mathbf{k}, \mathbf{k}^{\prime}}^{c}$ and $\mathcal{G}_{m \sigma, \mathbf{k}}^{0}$ the full and bare conduction electron Green's functions, respectively. [For a Kondo quantum dot tuned such that the low-energy physics is described by Eq. (1), the conductance $G$ through the dot has a form similar to Eq. (2), with $\rho$ replaced by $G .{ }^{29}$ ]

As mentioned in the Introduction, the ground state of the fully screened Kondo model is a spin singlet, and the regime of low-energy excitations below $T_{\mathrm{K}}$ shows Fermi-liquid behavior. ${ }^{13,15}$ One characteristic Fermiliquid property is that the leading dependence of the $T$ matrix on its arguments, when they are small relative to
$T_{\mathrm{K}}$, is quadratic,

$$
\frac{A_{m \sigma}(\varepsilon, T, B)}{A_{m \sigma}(0,0,0)}=1-\frac{c_{\varepsilon} \varepsilon^{2}+c_{T}^{\prime} T^{2}+c_{B} B^{2}}{T_{\mathrm{K}}^{2}},
$$

(Particle-hole and spin symmetries forbid terms linear in $\varepsilon$ or $B$.) This implies the same for the resistivity,

$$
\frac{\rho(T, B)}{\rho(0,0)}=1-\frac{c_{T} T^{2}+c_{B} B^{2}}{T_{\mathrm{K}}^{2}},
$$

with $c_{T}=\left(\pi^{2} / 3\right) c_{\varepsilon}+c_{T}^{\prime}$. The so-called Fermi-liquid coefficients $c_{\varepsilon}, c_{T}$ and $c_{B}$ are universal, $N$-dependent numbers, characteristic of the fully screened Fermi-liquid fixed point. For $N=1$, the coefficients $c_{T}$ and $c_{B}$ have recently been measured experimentally in transport studies through quantum dots and compared to theoretical predictions. ${ }^{39}$ The coefficient $c_{\varepsilon}$ is, in principle, also measurable via the non-linear conductance of a Kondo dot coupled strongly to one lead and very weakly to another. ${ }^{29}$ (The latter condition corresponds to the limit of a weak tunneling probe; it ensures that the non-linear conductance probes the equilibrium shape of the Kondo resonance, and hence the equilibrium Fermi-liquid coefficient $c_{\varepsilon}$.)

The goal of this paper is twofold: first, to analytically establish the $N$ dependence of $c_{\varepsilon}, c_{T}$ and $c_{B}$ using Fermiliquid theory similar to NB; and second, to numerically calculate them using an NRG code that exploits nonAbelian symmetries, in order to establish a benchmark for the quality of the latter. Our main results are as follows: First, if the Kondo temperature is defined by

$$
T_{\mathrm{K}}=\frac{N(N+2)}{3 \pi \chi^{\mathrm{imp}}}=\frac{4 S(S+1)}{3 \pi \chi^{\text {imp }}},
$$

where $\chi^{\text {imp }}$ is the static impurity susceptibility at zero temperature, the Fermi-liquid coefficients are given by

$$
c_{B}=\frac{(N+2)^{2}}{9}, \quad c_{T}=\pi^{2} \frac{4 N+5}{9}, \quad c_{\varepsilon}=\frac{2 N+7}{6} .
$$

For general $N$, the formula for $c_{T}$ has first been found by Yoshimori, ${ }^{21}$ while those for $c_{B}$ and $c_{\varepsilon}$ are new (though not difficult to obtain). Second, our numerical results for $N=1,2$ and 3 are found to agree with the predictions of Eq. (7) to within 5\%.

\section{FERMI-LIQUID THEORY}

In this section, we analytically calculate the Fermiliquid coefficients $c_{B}, c_{T}$ and $c_{\varepsilon}$ for general $N$. With the benefit of hindsight, we selectively combine various elements of the work on FLT of Nozières, ${ }^{13} \mathrm{NB},{ }^{15} \mathrm{AL}^{26}$ and $\mathrm{PG}^{29}$. Detailed justifications for the underlying assumptions are given by these authors in their original publications and hence will not be repeated here. Instead, our goal is to assemble their ideas in such a way that the route to the desired results is short and sweet. 
We begin by summarizing Nozières' ideas for expressing the $T$ matrix in terms of scattering phase shifts and expanding the latter in terms of phenomenological Fermiliquid parameters. Next, we recount AL's insight that this expansion can be reproduced systematically by doing perturbation theory in the leading irrelevant operator of the model's zero-temperature fixed point. Then we adopt PG's strategy of performing the expansion in a quasiparticle basis in which the contant part of the phase shift has already been taken into account, which considerably simplifies the calculation. Our own calculation is presented using notation analogous to that of $\mathrm{PG}$, while taking care to highlight the extra terms that arise for $N>1$. It turns out that their extra contributions can be found with very little extra effort.

\section{A. Phase shift and $T$ matrix}

Since the ground state of the fully screened Kondo model is a spin singlet, a low-energy quasiparticle scattering off the impurity experiences strong elastic scattering as if the impurity were nonmagnetic. Moreover, it also experiences a weak local interaction if some energy ( $\left.\ll T_{\mathrm{K}}\right)$ is available to weakly excite the singlet, causing some inelastic scattering. Since the singlet binding energy is $T_{\mathrm{K}}$, the strength of this local interaction is proportional to $1 / T_{\mathrm{K}}$.

Nozières ${ }^{13}$ realized that this combination of strong elastic scattering and a weak local interaction can naturally be treated in terms of scattering phase shifts. The phase shift of a quasiparticle with quantum numbers $m \sigma$ and excitation energy $\varepsilon$ relative to the Fermi energy can be written as

$$
\delta_{m \sigma}(\varepsilon)=\delta_{m \sigma}^{0}+\tilde{\delta}_{m \sigma}(\varepsilon), \quad \delta_{m \sigma}^{0}=\pi / 2 .
$$

Here $\delta_{m \sigma}^{0}$ is the phase shift for $\varepsilon=B=T=0$; it has the maximum possible value for scattering off a non-magnetic impurity, namely $\pi / 2$. Finite-energy corrections arising from weak excitations of the singlet are described by $\tilde{\delta}_{m \sigma}(\varepsilon)$, which is proportional to $1 / T_{\mathrm{K}}$.

If inelastic scattering is weak, unitarity of the $S$ matrix can be exploited ${ }^{13}$ to write the $T$ matrix in the following form (we use the notation $\mathrm{PG}^{29}$; for a detailed analysis, see AL's discussion ${ }^{26}$ of the terms arising from their Figs. 6 and 7 ):

$$
1-2 \pi \nu i \mathcal{T}_{m \sigma}(\varepsilon)=e^{2 i \delta_{m \sigma}(\varepsilon)}\left[1-2 \pi \nu i \tilde{\mathcal{T}}_{m \sigma}^{\mathrm{in}}(\varepsilon)\right] .
$$

Here $\tilde{\mathcal{T}}^{\text {in }}$ accounts for weak inelastic two-body scattering processes, and is proportional to $1 / T_{\mathrm{K}}^{2}$. It is to be calculated in a basis of quasiparticle states in which the phase shift $\delta_{m \sigma}^{0}$ has already been accounted for. (Here and below, tildes will be used on quantities defined with respect to the new basis if they differ from corresponding ones in the original basis.)

Expanding Eq. (9) in the small (real) number $\tilde{\delta}_{m \sigma}(\varepsilon)$ and recalling that $e^{2 i \delta_{m \sigma}^{0}}=-1$, one finds that the imaginary part of the $T$ matrix, which determines the spectral function, can be expressed as

$$
-\pi \nu \operatorname{Im} \mathcal{T}_{m \sigma}(\varepsilon)=1-\left[\tilde{\delta}_{m \sigma}^{2}(\varepsilon)-\pi \nu \operatorname{Im} \tilde{\mathcal{T}}_{m \sigma}^{\mathrm{in}}(\varepsilon)\right],
$$

to order $1 / T_{\mathrm{K}}^{2}$. Comparing this to Eq. (4), we conclude that knowing $\tilde{\delta}$ to order $1 / T_{\mathrm{K}}$ and $\tilde{\mathcal{T}}^{\text {in }}$ to order $1 / T_{\mathrm{K}}^{2}$ suffices to fully determine the Fermi-liquid coefficients $c_{B}, c_{T}$ and $c_{\varepsilon}$.

Now, a systematic calculation of $\tilde{\delta}$ and $\tilde{\mathcal{T}}^{\text {in }}$ requires a detailed theory for the strong-coupling fixed point, which became available only with the work of AL in the early 1990s. Nevertheless, Nozières succeeded in treating the case $N=1$ already in $1974,{ }^{13}$ using a phenomenological expansion of $\tilde{\delta}_{m \sigma}(\varepsilon)$ in powers of $\left(\varepsilon-\varepsilon_{\sigma}^{Z}\right) / T_{\mathrm{K}}\left[\varepsilon_{\sigma}^{\mathrm{Z}}\right.$ represents the Zeeman energy of quasiparticles in a magnetic field, see Eq. (14) below] and $\delta \bar{n}_{m^{\prime} \sigma^{\prime}}=n_{m^{\prime} \sigma^{\prime}}-n_{m^{\prime} \sigma^{\prime}}^{0}$, the deviation of the total quasiparticle number $n_{m^{\prime} \sigma^{\prime}}$ from its ground-state value. The prefactors in this expansion have the status of phenomenological Fermi-liquid parameters. Using various ingenious heuristic arguments, he was able to show that all these parameters, and also $\tilde{\mathcal{T}}^{\text {in }}$, are related to each other and can be expressed in terms of a single energy scale, namely the Kondo temperature. Moreover, by choosing the prefactor of $\varepsilon$ in this expansion to be $1 / T_{\mathrm{K}}$, he suggested a definition of the Kondo temperature that also fixes its numerical prefactor. (Our paper adopts this definition, too.) In 1980, NB generalized this strategy ${ }^{15}$ to general $N$, finding an expansion of the form

$$
\begin{aligned}
\tilde{\delta}_{m \sigma}(\varepsilon)= & \alpha\left(\varepsilon-\varepsilon_{\sigma}^{\mathrm{Z}}\right)-3 \psi \delta \bar{n}_{m,-\sigma} \\
& +\psi \sum_{m^{\prime} \neq m}\left(\delta \bar{n}_{m^{\prime} \sigma}-\delta \bar{n}_{m^{\prime},-\sigma}\right),
\end{aligned}
$$

where $\alpha$ and $\psi$ are phenomenological Fermi-liquid parameters related by $\alpha=3 \psi \nu=1 / T_{\mathrm{K}}$. [NB's initial version of Eq. (11) [their Eq. (34)] does not contain the Zeeman contribution $\varepsilon_{\sigma}^{Z}$, but the latter is implicit in their subsequent treatment of the Zeeman field before their Eq. (37).]

In the following subsections, we show how NB's expansion for $\tilde{\delta}$ can be derived systematically. $\mathrm{AL}^{26}$ and $\mathrm{PG}^{29}$ have shown how to do this for $N=1$; we will generalize their discussion to arbirtrary $N$.

\section{B. Leading irrelevant operator}

AL showed ${ }^{26}$ that NB's heuristic results can be derived in a systematic fashion by doing perturbation theory in the leading irrelevant operator of the model's zerotemperature fixed point. As perturbation, they took the operator with the lowest scaling dimension satisfying the requirements of being (i) local, (ii) independent of the impurity spin operator $\vec{S}$, since the latter is fully screened, (iii) $\mathrm{SU}(2)$-spin-invariant, (iv) and independent of the local charge density, just as the Kondo interaction. The operator sastifying these criteria has the form ${ }^{25}$

$$
H_{\lambda}=-\lambda: \vec{J}(0) \cdot \vec{J}(0):,
$$


where $\vec{J}(0)$ is the quasiparticle spin density at the impurity site, and $: \ldots$ : denotes the point-splitting regularization procedure (see Appendix). In Appendix D of Ref. 26, AL showed in great detail how NB's phase shifts can be computed using Eq. (12), for the single-channel case of $N=1$. They did not devote as much attention to the case of general $N$, though the needed generalizations are clearly implied in their work. We here present the corresponding calculation in some detail, following the notational conventions of $\mathrm{PG}$, which differ from those of AL in some regards (see Appendix). The main difference is that $\mathrm{PG}$ formulate the perturbation expansion in a new basis of quasiparticle states, in which the phase shift $\delta_{m \sigma}^{0}$ has already been accounted for, which somewhat simplifies the discussion. (We remark that PG chose $\delta_{m \sigma}^{0}=\sigma \pi / 2$ rather than $\pi / 2$ as used by $\mathrm{NB}$ and us, but the extra $\sigma$ has no consequences for the ensuing arguments.)

The quasiparticle Hamiltonian describing the vicinity of the strong-coupling fixed point (fp) has the form

$$
H_{\mathrm{fp}}=H_{\mathrm{fp}, 0}+H_{\lambda}
$$

where

$$
H_{\mathrm{fp}, 0}=\sum_{m \sigma k}\left(\xi_{k}+\varepsilon_{\sigma}^{\mathrm{Z}}\right): \psi_{k m \sigma}^{\dagger} \psi_{k m \sigma}:, \quad \varepsilon_{\sigma}^{\mathrm{Z}}=-\frac{\sigma B}{2}
$$

describes free quasiparticles in a magnetic field $B$, with Zeeman energy $\varepsilon_{\sigma}^{Z}$. Note that although the Zeeman term in the bare Hamiltonian (1) is local, it is global in Eq. (14), because the effective quasiparticle Hamiltonian $H_{\text {fp }}$ contains no local spin. Using standard point-splitting techniques, which we review in pedagogical detail in the Appendix, the leading irrelevant operator (12) can be written as $H_{\lambda}=H_{1}+H_{2}+H_{3}$, with

$$
\begin{aligned}
& H_{1}=-\frac{1}{2 \pi \nu T_{\mathrm{K}}} \sum_{m \sigma k k^{\prime}}\left(\xi_{k}+\xi_{k^{\prime}}\right): \psi_{k m \sigma}^{\dagger} \psi_{k^{\prime} m \sigma}:, \\
& H_{2}=\frac{1}{\pi \nu^{2} T_{\mathrm{K}}} \sum_{m}: \rho_{m \uparrow} \rho_{m \downarrow}: \\
& H_{3}=-\frac{2}{3 \pi \nu^{2} T_{\mathrm{K}}} \sum_{m \neq m^{\prime}}: \vec{j}_{m} \cdot \vec{j}_{m^{\prime}}:
\end{aligned}
$$

where

$$
\begin{aligned}
\rho_{m \sigma} & =\sum_{k k^{\prime} \sigma} \psi_{k m \sigma}^{\dagger} \psi_{k^{\prime} m \sigma} \\
\vec{j}_{m} & =\frac{1}{2} \sum_{k k^{\prime} \sigma \sigma^{\prime}} \psi_{k m \sigma}^{\dagger} \vec{\tau}_{\sigma \sigma^{\prime}} \psi_{k^{\prime} m \sigma^{\prime}}
\end{aligned}
$$

Here we have expressed the coupling constant $\lambda$ in terms of the inverse Kondo temperature using [cf. Eq. (A.11)]

$$
\lambda=\frac{8 \pi\left(\hbar v_{\mathrm{F}}\right)^{2}}{3 T_{\mathrm{K}}}
$$

with the numerical proportionality factor chosen such that $T_{\mathrm{K}}$ agrees with definition of the Kondo temperature used by NB and PG, as discussed below. Importantly, the point-splitting procedure fixes the relative prefactors

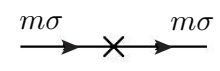

(a)

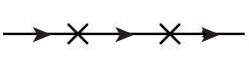

(d)

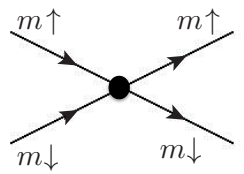

(b)

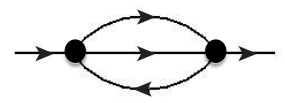

(e)

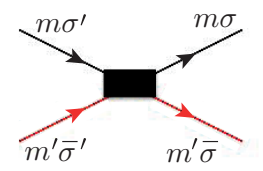

(c)

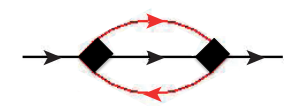

(f)
FIG. 1. (Color online) (a)-(c) Vertices associated with $H_{1}$, $\mathrm{H}_{2}$ and $H_{3}$, respectively. (d)-(f) Nonzero second-order contributions to the quasiparticle self-energy, $\tilde{\Sigma}_{m \sigma}^{R}$, involving $H_{1}^{2}$, $H_{2}^{2}$ and $H_{3}^{2}$, respectively. The contributions involving $H_{1} H_{2}$, $\mathrm{H}_{1} \mathrm{H}_{3}$ and $\mathrm{H}_{2} \mathrm{H}_{3}$ all vanish, the former two due to the odd power of energy in the two-leg vertex.

arising in $H_{1}, H_{2}$ and $H_{3}$ (whereas NB's approach requires heuristic arguments to fix them). Our notation for $H_{1}$ and $H_{2}$ coincides with that used by PG. $H_{3}$ contains all new contributions that enter additionally for $N>1$. Figure 1 gives a diagrammtic depiction of all three terms.

\section{First order terms}

Our first goal is to recover NB's expansion of the phase shift $\tilde{\delta}$ to leading order in $\varepsilon-\varepsilon_{\sigma}^{\mathrm{Z}}$ and $\delta \bar{n}$. Following PG, this can be done by calculating $\tilde{\delta}$ perturbatively to first order order in $1 / T_{\mathrm{K}}$, in the new basis of quasiparticle states that already incorporate the phase shift $\delta^{0}$. To order $1 / T_{\mathrm{K}}$, no inelastic scattering occurs, and $\tilde{\delta}$ is related to the elastic $T$ matrix by

$$
e^{2 i \tilde{\delta}_{m \sigma}(\varepsilon)}=1-2 \pi \nu i \tilde{\mathcal{T}}_{m \sigma}^{\mathrm{el}}(\varepsilon) .
$$

The elastic $T$ matrix, in turn, equals the real part of the quasiparticle self-energy, $\tilde{\mathcal{T}}_{m \sigma}^{\mathrm{el}}(\varepsilon)=\operatorname{Re} \tilde{\Sigma}_{m \sigma}^{R}(\varepsilon)$. (Actually, to order $1 / T_{\mathrm{K}}$, the self-energy is purely real.) By expanding Eq. (18) for small $\tilde{\delta}$, the phase shift is thus seen to be given by the real part of the self-energy:

$$
\tilde{\delta}_{m \sigma}(\varepsilon) \simeq-\pi \nu \operatorname{Re} \tilde{\Sigma}_{m \sigma}^{R}(\varepsilon) .
$$

Now, as pointed out already by Nozières in $1974,{ }^{13}$ a first-order perturbation calculation of the self-energy is equivalent to treating interaction terms in the mean-field (MF) approximation. They then take the form

$$
\begin{aligned}
& H_{2}^{\mathrm{MF}}=\frac{1}{\pi \nu^{2} T_{\mathrm{K}}} \sum_{m \sigma}: \rho_{m \sigma}: \delta \bar{n}_{m,-\sigma} \\
& H_{3}^{\mathrm{MF}}=-\frac{1}{3 \pi \nu^{2} T_{\mathrm{K}}} \sum_{\sigma} \sum_{m \neq m^{\prime}}: \rho_{m \sigma}:\left(\delta \bar{n}_{m^{\prime} \sigma}-\delta \bar{n}_{m^{\prime},-\sigma}\right),
\end{aligned}
$$


where $\delta \bar{n}_{m \sigma}=\left\langle: \rho_{m \sigma}:\right\rangle$, the quasiparticle number relative to the $B=0$ ground state, is given by

$$
\delta \bar{n}_{m \sigma}=-\nu \varepsilon_{\sigma}^{\mathrm{Z}}=\sigma \nu B / 2 .
$$

The mean-field version of the leading irrelevant operator thus has the form

$$
\begin{aligned}
H_{\lambda}^{\mathrm{MF}}=\sum_{m \sigma k k^{\prime}} h_{m \sigma}\left(\xi_{k}, \xi_{k^{\prime}}\right): \psi_{k m \sigma}^{\dagger} \psi_{k^{\prime} m \sigma}: \\
h_{m \sigma}\left(\xi_{k}, \xi_{k^{\prime}}\right)=\frac{1}{\pi \nu T_{\mathrm{K}}}\left[-\frac{1}{2}\left(\xi_{k}+\xi_{k^{\prime}}\right)+\frac{\delta \bar{n}_{m,-\sigma}}{\nu}\right. \\
\left.-\sum_{m^{\prime} \neq m} \frac{\delta \bar{n}_{m^{\prime} \sigma}-\delta \bar{n}_{m^{\prime},-\sigma}}{3 \nu}\right] .
\end{aligned}
$$

For such a single-particle perturbation, the self-energy can be directly read off from $h_{m \sigma}$ using

$$
\tilde{\Sigma}_{m \sigma}^{R}(\varepsilon)=h_{m \sigma}\left(\varepsilon-\varepsilon_{\sigma}^{\mathrm{Z}}, \varepsilon-\varepsilon_{\sigma}^{\mathrm{Z}}\right),
$$

because $k$ sums of the type $\sum_{k} 1 /\left(\varepsilon-\xi_{k}-\varepsilon_{\sigma}^{Z}+i 0^{+}\right)$yield residues involving $\xi_{k}=\varepsilon-\varepsilon_{\sigma}^{Z}$. Using Eq. (24) in Eq. (19) for the phase shift, we find

$$
\begin{aligned}
\tilde{\delta}_{m \sigma}(\varepsilon)=\frac{1}{T_{\mathrm{K}}}[\varepsilon & -\varepsilon_{\sigma}^{\mathrm{Z}}-\frac{\delta \bar{n}_{m,-\sigma}}{\nu} \\
& \left.+\sum_{m^{\prime} \neq m} \frac{\delta \bar{n}_{m^{\prime} \sigma}-\delta \bar{n}_{m^{\prime},-\sigma}}{3 \nu}\right] .
\end{aligned}
$$

This fully agrees with the expansion (11) of NB if we make the identification $1 / T_{\mathrm{K}}=\alpha=3 \psi \nu$, thus confirming the validity of NB's heuristic arguments. Note that the coefficient of $\varepsilon-\varepsilon_{\sigma}^{\mathrm{Z}}$ in Eq. (25) comes out as $1 / T_{\mathrm{K}}$, in agreement with the conventions of NB and PG, as intended by our choice of numerical prefactor in Eq. (17).

As consistency check, let us review how NB used Eq. (25) to calculate the Wilson ratio. First, Eq. (25) implies an impurity-induced change in the density of states per spin and channel of $\nu_{m \sigma}^{\mathrm{imp}}(\varepsilon)=\frac{1}{\pi} \partial_{\varepsilon} \delta_{m \sigma}(\varepsilon)$. This yields a corresponding impurity-induced change in the specific heat, $C^{\mathrm{imp}}$. At zero field (where $\varepsilon_{\sigma}^{\mathrm{Z}}$ and $\delta \bar{n}_{m \sigma}$ vanish), the change relative to the bulk is given by

$$
\frac{C^{\mathrm{imp}}}{C}=\frac{2 N \nu_{m \sigma}^{\mathrm{imp}}(0)}{2 N \nu}=\frac{1}{\pi \nu T_{\mathrm{K}}} .
$$

Second, the Friedel sum rule for the impurity-induced change in local charge in channel $m$ for spin $\sigma$ at $T=0$ gives

$$
N_{m \sigma}^{\mathrm{imp}}=\frac{1}{\pi} \delta_{m \sigma}(0)=\frac{1}{2}+\frac{1}{\pi} \tilde{\delta}_{m \sigma}(0),
$$

and Eq. (25), together with Eq. (21) for $\delta \bar{n}_{m \sigma}$, leads to

$$
\tilde{\delta}_{m \sigma}(0)=\frac{\sigma B}{T_{\mathrm{K}}}\left[\frac{1}{2}+\frac{1}{2}+\frac{N-1}{3}\right]=\frac{\sigma B(N+2)}{3 T_{\mathrm{K}}} .
$$

The linear response of the impurity-induced magnetization, $M^{\mathrm{imp}}=\frac{1}{2} \sum_{m}\left(N_{m \uparrow}^{\mathrm{imp}}-N_{m \downarrow}^{\mathrm{imp}}\right)$, then gives the impurity contribution to the spin susceptibility as

$$
\chi^{\mathrm{imp}}=\frac{M^{\mathrm{imp}}}{B}=\frac{N(N+2)}{3 \pi T_{\mathrm{K}}}=\frac{4 S(S+1)}{3 \pi T_{\mathrm{K}}} .
$$

(For all expressions involving $\chi^{\text {imp }}$ here and below, the limit $B \rightarrow 0$ is implied.) The corresponding bulk contribution is $\chi=\nu N / 2$. Thus, the Wilson ratio is found to be

$$
R=\frac{\chi^{\mathrm{imp}} / \chi}{C^{\mathrm{imp} / C}}=\frac{2(N+2)}{3}=\frac{4(S+1)}{3},
$$

in agreement with more elaborate calculations by Yoshimori $^{21}$ and by Mihály and Zawadowski. ${ }^{22}$

Note that Eq. (29) relates Nozières' definition of the Kondo temperature to an observable quantity, $\chi^{\mathrm{imp}}$, that can be calculated numerically. We used this as a precise way of defining $T_{\mathrm{K}}$ in our numerical work. (Subtleties involved in calculating $\chi^{\text {imp }}$ are discussed in Sec. IV B.) Note that up to a prefactor, Eq. (29) for $\chi^{\text {imp }}$ has the form $\chi^{\text {free }}\left(T_{\mathrm{K}}\right)$, where $\chi^{\text {free }}(T)=S(S+1) /(3 T)$ is the static susceptibility of a free spin $S$ at temperature $T$.

We are now in a position to extract our first Fermiliquid coefficient, $c_{B}$. For this, it suffices to know the spectral function $A$ in Eq. (4) to quadratic order in $B$, at $\varepsilon=T=0$, where $\tilde{\mathcal{T}}^{\text {in }}=0$. Inserting the corresponding expression (28) for $\tilde{\delta}_{m \sigma}(0)$ into Eq. (10) for $\operatorname{Im} \mathcal{T}$, we find

$$
A_{m \sigma}(0,0, B)=\frac{1}{\nu \pi^{2}}\left[1-\frac{(N+2)^{2}}{9} \frac{B^{2}}{T_{\mathrm{K}}^{2}}\right] \text {. }
$$

Comparing this to Eq. (4), we read off $c_{B}=(N+2)^{2} / 9$.

Note that if the definition (29) of $T_{\mathrm{K}}$ in terms of $\chi^{\text {imp }}$ is taken as given, $c_{B}$ can actually be derived on the back of an envelope: for a fully screened Kondo model, the impurity-induced spin susceptibility gets equal contributions from all $N$ channels, $\chi^{\mathrm{imp}}=N \chi_{m}^{\mathrm{imp}}$, and the Friedel sum rule relates the contribution from each channel to phase shifts, $\chi_{m}^{\mathrm{imp}}=M_{m}^{\mathrm{imp}} / B=\left[\tilde{\delta}_{m \uparrow}(0)-\tilde{\delta}_{m \downarrow}(0)\right] /(2 \pi B)$, implying $\tilde{\delta}_{m \sigma}(0)=\sigma\left(\pi \chi^{\mathrm{imp}} / N\right) B$. Using this in Eq. (10) yields

$$
A_{m \sigma}(0,0, B)=\frac{1}{\nu \pi^{2}}\left[1-\left(\pi \chi^{\mathrm{imp}} / N\right)^{2} B^{2}\right]
$$

which is equivalent to Eq. (31) if Eq. (29) holds.

\section{Second order terms}

We next discuss inelastic scattering for $B=0$, but at finite temperature. To order $1 / T_{\mathrm{K}}^{2}$, inelastic scattering is described by the imaginary part of the quasiparticle self-energy arising from the second-order contributions of $H_{1}, H_{2}$ and $H_{3}$, shown in diagrams (d)-(f) of Fig. 1, 
respectively. These diagrams give

$$
\begin{aligned}
\operatorname{Im} \tilde{\Sigma}_{m \sigma}^{R, 1}(\varepsilon) & =-\frac{\varepsilon^{2}}{\pi \nu T_{\mathrm{K}}^{2}}, \\
\operatorname{Im} \tilde{\Sigma}_{m \sigma}^{R, 2}(\varepsilon) & =-\frac{\varepsilon^{2}+\pi^{2} T^{2}}{2 \pi \nu T_{\mathrm{K}}^{2}}, \\
\operatorname{Im} \tilde{\Sigma}_{m \sigma}^{R, 3}(\varepsilon) & =\frac{2}{3}(N-1) \operatorname{Im} \tilde{\Sigma}_{m \sigma}^{R, 2}(\varepsilon) .
\end{aligned}
$$

The first two can also be found in the discussion of PG, whose strategy we follow here. (They also appear, in slightly different guise, in the discussion of $\mathrm{AL}^{26}$.) The third is proportional to the second, and the factor $2 / 3$ originates from $(2 / 3)^{2} 2 s(s+1)$ with $s=1 / 2$, since the relative prefactor between $\mathrm{H}_{3}$ and $\mathrm{H}_{2}$ brings in two powers of $2 / 3$, and the algebra of Pauli matrices yields a factor $2 s(s+1)$.

Now, the term called $\tilde{\mathcal{T}}^{\text {in }}$ in Eq. (9) by definition describes the contribution of the two-body terms $\mathrm{H}_{2}$ and $\mathrm{H}_{3}$ to inelastic scattering:

$$
\operatorname{Im} \tilde{\mathcal{T}}_{m \sigma}^{\text {in }}(\varepsilon)=\operatorname{Im}\left[\tilde{\Sigma}_{m \sigma}^{R, 2}(\varepsilon)+\tilde{\Sigma}_{m \sigma}^{R, 3}(\varepsilon)\right] .
$$

The contribution $\operatorname{Im} \tilde{\Sigma}^{R, 1}$ from $H_{1}$ is not included in $\operatorname{Im} \tilde{\mathcal{T}}^{\text {in }}$ here, since it actually equals $-\tilde{\delta}^{2} / \pi \nu$, and hence is already contained in the factor $e^{2 i \tilde{\delta}}$ in Eq. (9). Indeed, in Eq. (10) for the imaginary part of the $T$ matrix in the original basis, the $\tilde{\delta}^{2}$ term equals $-\pi \nu \operatorname{Im} \tilde{\Sigma}^{R, 1}$. Collecting all ingredients, Eq. (10) gives

$$
\begin{aligned}
& A_{m \sigma}(\varepsilon, T, 0) \\
& =\frac{1}{\nu \pi^{2}}\left[1-\frac{\varepsilon^{2}}{T_{\mathrm{K}}^{2}}-\frac{\varepsilon^{2}+\pi^{2} T^{2}}{2 T_{\mathrm{K}}^{2}}\left(1+\frac{2}{3}(N-1)\right)\right] \\
& =\frac{1}{\nu \pi^{2}}\left[1-\frac{(2 N+7) \varepsilon^{2}+(2 N+1) \pi^{2} T^{2}}{6 T_{\mathrm{K}}^{2}}\right] .
\end{aligned}
$$

For $N=1$, the second term reduces to the familiar form $-\left(3 \varepsilon^{2}+\pi^{2} T^{2}\right) /\left(2 T_{\mathrm{K}}^{2}\right)$ found by $\mathrm{AL}^{26}$ and $\mathrm{GP}^{29}$. Comparing Eqs. (35) and (4) and (5) we read off $c_{\varepsilon}=(2 N+7) / 6$ and $c_{T}^{\prime}=\pi^{2}(2 N+1) / 6$, implying $c_{T}=\pi^{2}(4 N+5) / 9$.

\section{NRG RESULTS}

In this section, we describe our NRG work. We had set ourselves the goal of achieving an accuracy of better than $5 \%$ for the Fermi-liquid coefficients. To achieve this, two ingredients were essential. First, exploiting non-Abelian symmetries; and second, defining the Kondo temperature with due care. The latter is a matter of some subtlety ${ }^{34}$ because the wide-band limit assumed in analytical work does not apply in numerical calculations.

We begin below by giving the Lehmann representation for the desired spectral function. We then discuss the non-Abelian symmetries used in our NRG calculations and explain how the Kondo temperature was extracted numerically. Finally, we present our numerical results.

\section{A. NRG details}

To numerically calculate the $T$ matrix of Eq. (3), we use equations of motion ${ }^{37,38}$ to express it as

$$
\begin{aligned}
\mathcal{T}_{m \sigma}(\varepsilon) & =J_{\mathrm{K}}\left\langle S_{z}\right\rangle+\left\langle\left\langle O_{m \sigma} ; O_{m \sigma}^{\dagger}\right\rangle\right\rangle, \\
O_{m \sigma} & \equiv\left[\Psi_{m \sigma}(0), H_{\mathrm{loc}}\right]=J_{\mathrm{K}} \sum_{\sigma^{\prime}} \vec{S} \cdot \frac{\vec{\tau}_{\sigma \sigma^{\prime}}}{2} \Psi_{m \sigma^{\prime}}(0) .
\end{aligned}
$$

Here $\langle\langle\cdot ; \cdot\rangle\rangle$ denotes a retarded correlation function, and $\Psi_{m \sigma}(0)=\frac{1}{\sqrt{N_{\text {disc }}}} \sum_{k} c_{k m \sigma}$, where $N_{\text {disc }}$ is the number of discrete levels in the band (and hence proportional to the system size). The spectral function is then calculated in its Lehmann-representation,

$$
\begin{aligned}
& A_{m \sigma}(\varepsilon, T, B)= \\
& \quad \sum_{a, b} \frac{e^{-\beta E_{a}}+e^{-\beta E_{b}}}{Z}\left|\left\langle a\left|O_{m \sigma}\right| b\right\rangle\right|^{2} \delta\left(\varepsilon-E_{a b}\right),
\end{aligned}
$$

with $E_{a b}=E_{b}-E_{a}$, using the full density matrix (FDM) approach of NRG. ${ }^{9,40-42}$

For our numerical work, we take the conduction band energies to lie within the interval $\xi_{k} \in[-D, D]$, with Fermi energy at 0 and half-bandwidth $D=1$, and take the density of states per spin, channel and unit length to be constant, as $1 / 2 D$. (It is related to the extensive density of states used in Sec. III by $\nu=N_{\text {disc }} / 2 D$.) For the calculations used to determine the Fermi-liquid parameters, we use exchange coupling $\nu J_{\mathrm{K}}=0.1$, so that the Kondo temperature $T_{\mathrm{K}} / D \propto \exp \left[-1 /\left(\nu J_{\mathrm{K}}\right)\right]$ has the same order of magnitude for $N=1,2$ and 3, namely $\lesssim 10^{-4}$. Following standard NRG protocol, ${ }^{7,8,10}$ the conduction band is discretized logarithmically with discretization parameter $\Lambda$, mapped onto a Wilson chain, and diagonalized iteratively. NRG truncation at each iteration step is controlled by either specifying the number of kept states per shell, $N_{\mathrm{K}}$, or the truncation energy, $E_{\mathrm{tr}}$ (in rescaled units, as defined in Ref. 43), corresponding to the highest kept energy per shell. Spectral data are averaged over $N_{z}$ different, interleaving logarithmic discretization meshes. ${ }^{44}$ The values for NRG-specific parameters used here are given in legends in the figures below.

For the fully screened $N$-channel Kondo model, the dimension of the local Hilbert space of each supersite of the Wilson chain is $4^{N}$. Since this increases exponentially with the number of channels, it is essential, specifically so for $N=3$, to reduce computational costs by exploiting non-Abelian symmetries ${ }^{11}$ to combine degenerate states into multiplets. Several large symmetries are available ${ }^{19}$ : For $B=0$, the model has $\mathrm{SU}(2) \times \mathrm{U}(1) \times \mathrm{SU}(N)$ spincharge-channel symmetry. If the bands desribed by $H_{0}$ are particle-hole symmetric, as assumed here, the model also has a $\mathrm{SU}(2) \times[\mathrm{SU}(2)]^{N}$ spin-(charge) ${ }^{N}$ symmetry, involving $\mathrm{SU}(2)$ mixing of particles and holes in each of the $N$ channels. The $\mathrm{U}(1) \times \mathrm{SU}(N)$ and $[\mathrm{SU}(2)]^{N}$ symmetries are not mutually compatible (their generators do not all commute), however, implying that both are subgroups of a larger symmetry group, the symplectic 
$\operatorname{Sp}(2 N)$. Thus the full symmetry of the model for $B=0$ is $\mathrm{SU}(2) \times \mathrm{Sp}(2 N)$. For $B \neq 0$ it is $\mathrm{U}(1) \times \operatorname{Sp}(2 N)$, since a finite magnetic field breaks the $\mathrm{SU}(2)$ spin symmetry to the Abelian U(1) $S_{z}$ symmetry. When the model's full symmetry is exploited, the multiplet spaces encountered in NRG calculations exhibit no more degeneracies in energy at all.

Using only Abelian symmetries turned out to be clearly insufficient to obtain well converged numerical data for $N=3$, despite having a relatively large $\Lambda$. This, however, is required for accurate Fermi-liquid coefficients with errors below a few percent. For numerically converged data, therefore, it was essential to use non-Abelian symmetries. For our $B=0$ calculations, it turned out to be sufficient to use $\mathrm{SU}(2) \times \mathrm{U}(1) \times \mathrm{SU}(N)$ symmetry for calculating $c_{T}$, but the full $\mathrm{SU}(2) \times \mathrm{Sp}(2 N)$ symmetry was needed for calculating $c_{\varepsilon}$. Likewise, for our $B \neq 0$ calculations of $c_{B}$, we needed to use the full $\mathrm{U}(1) \times \operatorname{Sp}(2 N)$ symmetry. Doing so led to an enormous reduction in memory requirements, the more so the larger the rank of the symmetry group $[\operatorname{Sp}(2 N)$ has $\operatorname{rank} N$, and $\mathrm{SU}(N)$ has rank $N-1]$. For $N=3$, for example, we kept $\lesssim 13500$ multiplets for $\mathrm{SU}(2) \times \mathrm{U}(1) \times \mathrm{SU}(3)$ or $\lesssim 3357$ multiplets for $\mathrm{SU}(2) \times \mathrm{Sp}(6)$ during $\mathrm{NRG}$ truncation, which, in effect, amounts to keeping $\lesssim 980000$ individual states. ${ }^{11}$

\section{B. Definition of $T_{\mathrm{K}}$}

The Fermi-liquid theory of Sec. III implicitly assumes that the model is considered in the so-called scaling limit, in which the ratio of Kondo temperature to bandwidth vanishes, $T_{\mathrm{K}} / D \rightarrow 0$. In this limit, physical quantities such as $\rho(T, B) / \rho(0,0)$ are universal scaling functions, which depend on their arguments only in the combinations $B / T_{\mathrm{K}}$ and $T / T_{\mathrm{K}}$. Since the shape of such a scaling function, say $\rho(0, B) / \rho(0,0)$ plotted versus $B / T_{\mathrm{K}}$, is universal, i.e. independent of the bare parameters (coupling $J_{\mathrm{K}}$ and bandwidth $D$ ) used to calculate it, curves generated by different combinations of bare parameters can all be made to collapse onto each other by suitably adjusting the parameter $T_{\mathrm{K}}$ for each. In the same sense the Fermiliquid parameters $c_{B}, c_{T}$ and $c_{\varepsilon}$, being Taylor-coefficients of universal curves, are universal, too.

One common way to achieve a scaling collapse, popular particularly in experimental studies, is to identify the Kondo temperature with the field $B_{1 / 2}$ or temperature $T_{1 / 2}$ at which the impurity contribution to the resisitivity has decreased to half its unitary value,

$$
\rho\left(0, B_{1 / 2}\right)=\rho(0,0) / 2, \quad \rho\left(T_{1 / 2}, 0\right)=\rho(0,0) / 2 .
$$

However, this is approach is not suitable for the purpose of extracting Fermi-liquid coefficients, for which $T_{\mathrm{K}}$ has to be defined in terms of (analytically accessible) lowenergy properties characteristic of the strong-coupling fixed point. In Sec. III we have therefore adopted Nozières' definition of $T_{\mathrm{K}}$ in terms of the leading energy dependence of the phase shift $\tilde{\delta}_{m \sigma}^{0}$ [Eq. (25)], implying that it can be expressed in terms of $\chi^{\text {imp }}$, of the local static spin susceptibility at zero temperature [Eq. (29)]. In the scaling limit, this definition of $T_{\mathrm{K}}$ matches $B_{1 / 2}$ or $T_{1 / 2}$ up to prefactors, i.e. $B_{1 / 2} / T_{\mathrm{K}}$ and $T_{1 / 2} / T_{\mathrm{K}}$ are universal, $N$-dependent numerical constants, independent of the model's bare parameters.

In numerical work, however, the scaling limit is never fully realized, since the bandwidth is always finite. It may thus happen that a scaling collapse expected analytically is not found when the corresponding curves are calculated numerically. For example, if the Kondo temperature is defined, as seems natural, in terms of a purely local susceptibility, $\chi^{\mathrm{loc}}$, involving only the response of the local spin to a local field,

$$
\left.\frac{4 S(S+1)}{3 \pi T_{\mathrm{K}}^{\mathrm{loc}}} \equiv \chi^{\mathrm{loc}} \equiv \frac{d}{d B}\left\langle S_{z}\right\rangle\right|_{B=0},
$$

then curves expected to show a scaling collapse actually do not collapse onto each other, as pointed out recently in Ref. 34 (see Figs. 2(d)-(f) there). That paper also showed how to remedy this problem: the static spin susceptibility used to calculate $T_{\mathrm{K}}$ has to be defined more carefully, and two slightly different definitions have to be used, depending on the context. The first option is needed when studying zero-temperature (i.e. ground state) properties as a function of some external parameter, such as the field dependence of the resisitivity (needed for $c_{B}$ ). In this case, a corresponding susceptibility defined in terms of the response of the system's total spin to a local field should be used:

$$
\left.\frac{4 S(S+1)}{3 \pi T_{\mathrm{K}}^{\mathrm{FS}}} \equiv \chi^{\mathrm{FS}} \equiv \frac{d}{d B}\left\langle S_{z}^{\mathrm{tot}}\right\rangle\right|_{B=0} .
$$

The superscript FS stands for "Friedel sum rule", to highlight the fact that using this rule to calculate the linear response of $\left\langle S_{z}^{\text {tot }}\right\rangle$ to a local field directly leads to relation (29) between $\chi^{\mathrm{imp}}$ and $T_{\mathrm{K}}$. The second option is needed when studying dynamical or thermal quantities that depend on the system's many-body excitations for given fixed external parameters (e.g. fixed $B=0$ ), such as the temperature-dependence of the resistivity (needed for $c_{T}$ ), or the curvature of the Kondo resonance (needed for $c_{\varepsilon}$ ). In this case, one should use

$$
\frac{4 S(S+1)}{3 \pi T_{\mathrm{K}}^{\mathrm{sc}}} \equiv \chi^{\mathrm{sc}} \equiv 2 \chi^{\mathrm{FS}}-\chi^{\mathrm{loc}} .
$$

The superscript sc stands for "scaling", to indicate that this definition of the Kondo temperature ensures ${ }^{34}$ a scaling collapse of dynamical or thermal properties. Figure 2 demonstrates that a scaling collapse is indeed found when the field- or temperature-dependent resistivity, plotted versus $B / T_{\mathrm{K}}^{\mathrm{FS}}$ or $T / T_{\mathrm{K}}^{\mathrm{sc}}$, respectively, is calculated for two different values of $J_{\mathrm{K}}$ (solid and dashed lines, respectively). Note that this works equally well for $N=1,2$ and 3. (For $N=1$, such scaling collapses had already been shown in Ref. 34.) 

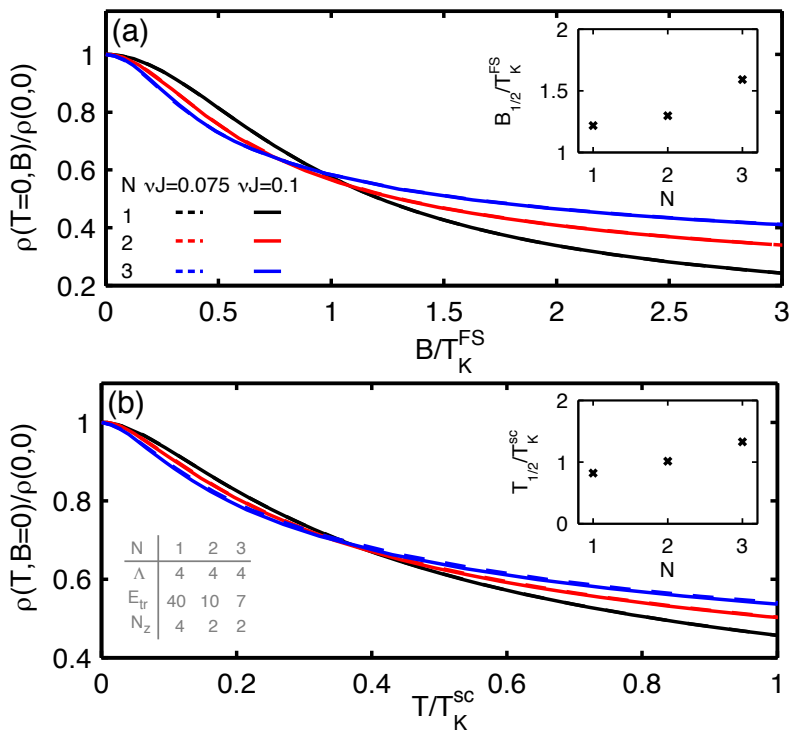

FIG. 2. (Color online) Scaling collapse of (a) the resistivity at zero temperature as function of field, and (b) at zero field as function of temperature, calculated for two different values of the bare coupling, $\nu J_{\mathrm{K}}$ (dashed or solid), and for $N=$ 1,2 and 3 . For each $N$, the dashed and solid curves overlap so well that they are almost indistinguishable. The insets compare the energy scales $B_{1 / 2}$ and $T_{1 / 2}$ at which the resistivity has decreased to half its unitary value [cf. Eq. (38)], to the scales $T_{\mathrm{K}}^{\mathrm{FS}}$ and $T_{\mathrm{K}}^{\mathrm{sc}}$ [cf. Eqs. (40) and (41)], respectively. The shown ratios are universal numbers of order unity, but not necessarily very close to 1 , with a significant dependence on $N: B_{1 / 2} / T_{\mathrm{K}}^{\mathrm{FS}}=1.22,1.31,1.60$ and $T_{1 / 2} / T_{\mathrm{K}}^{\mathrm{sc}}=0.82,1.02,1.36$ for $N=1,2$ and 3 , respectively. The legend in the lower left of panel (b) specifies the NRG parameters used for both panels.

We remark that the three Kondo temperatures defined in Eqs. (39)-(41) differ quite significantly from each other for the Kondo Hamiltonian of Eq. (1), with differences as large as $12 \%, 31 \%$ and $55 \%$ for $N=1,2$ and 3 , respectively, for the parameters used in Fig. 2. This indicates that although we have chosen bare paramters for which $T_{\mathrm{K}} / D$ is smaller than $10^{-4}$, we have still not reached the scaling limit [in which the definitions Eq. (39)-(41) of the Kondo temperature should all coincide numerically $\left.{ }^{34}\right]$. We have checked that the differences between $T_{\mathrm{K}}^{\text {loc }}, T_{\mathrm{K}}^{\mathrm{FS}}$ and $T_{\mathrm{K}}^{\mathrm{sc}}$ decrease when $\nu J_{\mathrm{K}}$ is reduced in an attempt to get closer to the scaling limit, but estimate that truly reaching that limit would require $\nu J_{\mathrm{K}}<0.01$ for the Kondo model, implying $T_{\mathrm{K}} / D<10^{-45}$. Thus, reaching the scaling limit by brute force is numerically unfeasible. Therefore, using $T_{\mathrm{K}}^{\mathrm{FS}}$ and $T_{\mathrm{K}}^{\mathrm{sc}}$ rather than $T_{\mathrm{K}}^{\text {loc }}$ is absolutely essential for obtaining scaling collapses. It is similarly essential for an accurate determination of the Fermi-liquid parameters. Correspondingly, for the results discussed below, we have used $T_{\mathrm{K}}^{\mathrm{FS}}$ as definition of the Kondo temperature when extracting $c_{B}$, and $T_{\mathrm{K}}^{\mathrm{sc}}$ when extracting $c_{T}$ and $c_{\varepsilon}$.

\section{Using unbroadened discrete data only}

When one is interested in spectral properties, one typically has to broaden the discrete data. For the determination of the Fermi-liquid coefficients, however, where high numerical accuracy is required, it is desirable to avoid standard broadening. For the calculation of $c_{T}$ and $c_{B}$ this can be achieved ${ }^{9}$ by directly inserting the Lehmann sum over $\delta$ functions for the spectral function $A_{m \sigma}(\varepsilon, T, B)$ [Eq. (37)] into the energy integral for $\rho(T, B)[$ Eq. (2)], resulting in a sum over discrete data points that produces a smooth curve. The curve is smooth because Eq. (2) in effect thermally broadens the $\delta$ peaks in the Lehmann representation. This is true even in the limit $T \rightarrow 0$, because in NRG calculations it is realized by taking $T$ nonzero, but much smaller than all other energy scales.

For the determination of $c_{\varepsilon}$, in contrast, one faces the problem that $A_{m \sigma}(\varepsilon, 0,0)$ is represented not as an integral of a sum over discrete $\delta$ functions, but directly in terms of the latter. To avoid having to broaden these by hand, it is desirable to find a way to extract $c_{\varepsilon}$ from an expression involving an integral over the discrete spectral data, as for $c_{B}$ and $c_{T}$. This can be achieved as follows. First, note that $c_{\varepsilon}$ is, by definition, a coefficient in the general Taylor expansion of the normalized spectral function $A^{\mathrm{norm}}(\varepsilon) \equiv A_{m \sigma}(\varepsilon, 0,0) / A_{m \sigma}(0,0,0)$ for small frequencies,

$$
A^{\mathrm{norm}}(\varepsilon)=\sum_{n=0}^{\infty} a_{n}\left(\varepsilon / T_{\mathrm{K}}\right)^{n}, \quad c_{\varepsilon}=a_{2} .
$$

Due to particle-hole symmetry, $a_{n}=0$ for all $n$ odd, and by definition $a_{0}=1$. To determine $a_{2}$ from an integral over discrete data, we consider a weighted average of $A^{\text {norm }}(\varepsilon)$ over $\varepsilon$,

$$
\bar{A}(\tau) \equiv \int d \varepsilon A^{\mathrm{norm}}(\varepsilon) P_{\tau}(\varepsilon)
$$

where $P_{\tau}(\varepsilon)$ is a symmetric weighting function of width $\tau$ and weight 1 , and moments defined by

$$
\int d \varepsilon(\varepsilon / \tau)^{n} P_{\tau}(\varepsilon) \equiv p_{n}
$$

for integer $n \geq 0$ (with $p_{0}=1$ ). Here we use

$$
P_{\tau}(\varepsilon)=\frac{1}{4 \tau} \frac{1}{\cosh ^{2}(\varepsilon / 2 \tau)}=-\frac{\partial f(\varepsilon, \tau)}{\partial \varepsilon},
$$

but other choices are possible, too (e.g., a Gaussian peak). Clearly, the leading $\tau$ dependence of $\bar{A}(\tau)$ for small $\tau$ reflects the leading $\varepsilon$ dependence of $A^{\text {norm }}(\varepsilon)$ and allows for an accurate determination of $a_{2}$. Indeed, using Eqs. (42)-(45), we obtain a power-series expansion for $\bar{A}(\tau)$ of the form $\bar{A}(\tau)=\sum_{n} a_{n} p_{n}\left(\tau / T_{\mathrm{K}}\right)^{n}$. Thus, by fitting $\bar{A}^{\text {fit }}(\tau)=\sum_{n} f_{n} \tau^{n}$ to the NRG data for $\bar{A}(\tau)$, one can determine the desired coefficients in (42) using $a_{n}=T_{\mathrm{K}}^{n} f_{n} / p_{n}$. In particular, the Fermi-liquid coefficient of present interest is given by $c_{\varepsilon}=a_{2}=T_{\mathrm{K}}^{2} f_{2} / p_{2}$. 

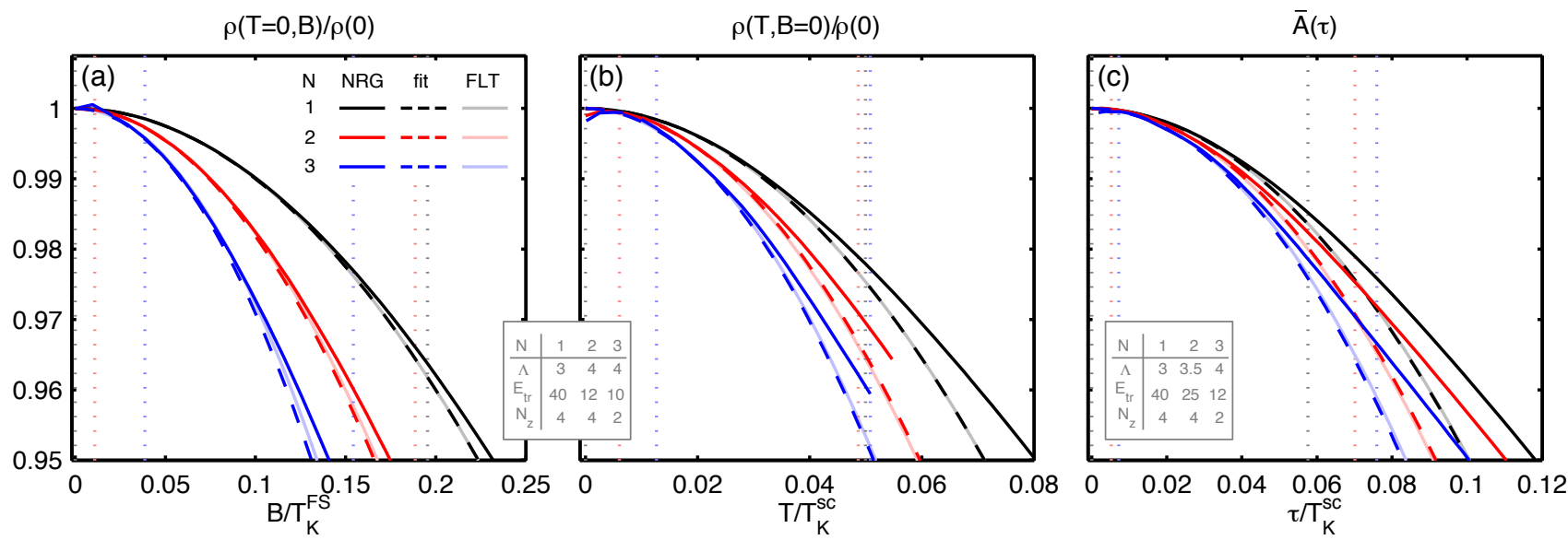

FIG. 3. (Color online) (a) Resistivity as function of magnetic field at $T=0$, (b) resistivity as function of temperature at $B=0$, and (c) the weighted spectral function $\bar{A}(\tau)$ [cf. Eq. (43)] at $T=B=0$, all shown for $N=1,2,3$. Each panel contains NRG data (heavy solid lines), the quadratic term from a fourth order polynomial fit (heavy dashed lines) and the corresponding predictions from FLT of Eq. (7) for the quadratic term (light solid lines). Left and right vertical dotted lines in matching colors indicate the lower and upper borders of the fitting range used for each $N$. The boxed legends specify the NRG parameters used here.

\section{Extraction of Fermi-liquid coefficients}

Figs. 3(a)-(c) show our NRG data (heavy solid lines) for the resistivity plotted versus $B / T_{\mathrm{K}}^{\mathrm{FS}}$ at zero temperature or plotted versus $T / T_{\mathrm{K}}^{\mathrm{sc}}$ at zero field, and for the weighted spectral function plotted versus $\tau / T_{\mathrm{K}}^{\mathrm{sc}}$, respectively. We determined the Fermi-liquid coefficients $c_{B}, c_{T}$ and $c_{\varepsilon}$ from the quadratic terms of fourth-order polynomial fits to these curves. Including the fourth-order term allows the fitting range to be extended towards somewhat larger values of the argument, thus increasing the accuracy of the fit. For each solid curve, the quadratic term from the fit is shown by heavy dashed lines; these are found to agree well with the corresponding predictions from FLT, shown by light lines of matching colors. The level of agreement is quite remarkable, given the rather limited range in which the behavior is purely quadratic: with increasing argument, quartic contributions become increasingly important, as reflected by the growing deviations between dashed and solid lines; and at very small values of the argument $(\lesssim 0.02)$, the NRG data become unreliable due to known NRG artefacts.

Numerical values for the extracted Fermi-liquid coefficients are given in Table I; they agree with those predicted analytically to within $\leq 5 \%$. This can be considered excellent agreement, especially for the numerically very challenging case of $N=3$.

\section{CONCLUSIONS}

Our two main results can be summarized as follows. First, we have presented a compact derivation of three Fermi-liquid coefficients for the fully-screened $N$-channel Kondo model, by generalizing well-established calcula-

\begin{tabular}{cccc}
\hline \hline$N$ & $c_{B}^{\mathrm{NRG}} / c_{B}^{\mathrm{FLT}}$ & $c_{T}^{\mathrm{NRG}} / c_{T}^{\mathrm{FLT}}$ & $c_{\varepsilon}^{\mathrm{NRG}} / c_{\varepsilon}^{\mathrm{FLT}}$ \\
\hline 1 & $1.00 \pm 0.01$ & $1.00 \pm 0.01$ & $1.01 \pm 0.03$ \\
2 & $1.02 \pm 0.03$ & $0.98 \pm 0.03$ & $0.99 \pm 0.03$ \\
3 & $1.05 \pm 0.05$ & $1.01 \pm 0.03$ & $1.02 \pm 0.07$ \\
\hline \hline
\end{tabular}

TABLE I. Numerically extracted values of $c_{B}, c_{T}$ and $c_{\varepsilon}$, given here relative to the corresponding predictions from FLT of Eq. (7). The deviations between NRG and FLT values are $\leq 5 \%$ in all cases. To numerically determine these coefficients, we used the quadratic coefficient of a fourth-order polynomial fit to the corresponding NRG data. Error bars were estimated by comparing the quartic fits to polynomial fits of different higher orders.

tions for $N=1$ to general $N$. The corresponding calculations, building on ideas of Nozières, Affleck and Ludwig, and Pustilnik and Glazman, are elementary. We hope that our way of presenting them emphasizes this fact, and perhaps paves the way for similar calculations in less trivial quantum impurity problems that also show Fermiliquid behavior, such as the asymmetric single-impurity Anderson Hamiltonian, or the 0.7-anomaly in quantum point contacts. ${ }^{45}$

Second, we have established a benchmark for the quality of NRG results for the fully screened $N$-channel Kondo model, by showing that it is possible to numerically calculate equilibrium Fermi-liquid coefficients with an accuracy of better than $5 \%$ for $N=1,2$ and 3 . To achieve numerical results of this quality, two technical ingredients were essential, both of which became available only recently: first, exploiting larger-rank non-Abelian 
symmetries in the numerics; ${ }^{11,12}$ and second, carefully defining the Kondo temperature ${ }^{34}$ in such a way that numerically-calculated universal scaling curves are indeed universal, in the sense of showing a proper scaling collapse, despite the fact that the scaling limit $T_{\mathrm{K}} / D \rightarrow 0$ is typically not achieved in numerical work.

\section{ACKNOWLEDGEMENTS}

We acknowledge helpful discussions with K. Kikoin, C. Mora, A. Ludwig and G. Zarànd. We are grateful to D. Schuricht for drawing our attention to Ref. 27, and for sending us a preprint of Ref. 46 prior to its submission. The latter work, which we received in the final stages of this work, also uses $H_{\lambda}$ of Eq. (12) as starting point for calculating Fermi-liquid coefficients for the $N$-channel Kondo model, and its result for $c_{T}$ is consistent with our own. We gratefully acknowledge financial support from the DFG (WE4819/1-1 for A.W., and SFB-TR12, SFB631 and the Cluster of Excellence Nanosystems Initiative Munich vor J.v.D., M.H., and A.W.)

\section{Appendix}

This appendix offers a pedagogical derivation of the Hamiltonian $H_{\lambda}$ given in Eq. (15) of the main text using the point-splitting regularization strategy, following AL (Appendix D of 26). Its main purpose is to show how the relation $\alpha=3 \psi \nu=1 / T_{\mathrm{K}}$ between Fermi-liquid parameters that NB had found by intuitive arguments ${ }^{15}$ follows simply and naturally from point splitting. For a detailed discussion of the point-splitting strategy, see Refs. 47-49.

According to $\mathrm{AL}$, the leading irrelevant operator for the fully screened $N$-channel Kondo model has the form

$$
H_{\lambda}=-\lambda: \vec{J}(0) \cdot \vec{J}(0): .
$$

Here $\vec{J}(x)=\sum_{m=1}^{N}: \vec{J}_{m}(x)$ : is the total (point-split) spin density from all channels at position $x$ (the impurity or dot sits at $x=0$ ), and

$$
\vec{J}_{m}(x)=\frac{1}{2} \sum_{\sigma \sigma^{\prime}} \Psi_{m \sigma}^{\dagger}(x) \vec{\tau}_{\sigma \sigma^{\prime}} \Psi_{m \sigma^{\prime}}(x)
$$

is the corresponding (non-point-split) spin density for channel $m$. Here : ... : denotes point splitting,

$$
: A(x) B(x): \equiv \lim _{\eta \rightarrow 0}[A(x+\eta) B(x)-A(x+\eta) B(x)],
$$

a field-theoretic scheme for regularizing products of operators at the same point by subtracting their ground state expecation value, $A B=\langle A B\rangle$. (In most cases, point splitting is equivalent to normal ordering.) For present purposes, we follow $\mathrm{AL}^{26}$ and take

$$
\Psi_{m \sigma}(x)=\frac{1}{\sqrt{L}} \sum_{k} e^{-i k x} \psi_{k m \sigma}
$$

to be free fermion fields with linear dispersion $\left(\xi_{k}=\right.$ $k \hbar v_{\mathrm{F}}$ ) in a box of length $L \rightarrow \infty$ (with $k \in 2 \pi n / L, n \in$ $\mathbb{Z})$, with normalization $\left\{\psi_{k m \sigma}, \psi_{k^{\prime} m^{\prime} \sigma^{\prime}}^{\dagger}\right\}=\delta_{k k^{\prime}} \delta_{m m^{\prime}} \delta_{\sigma \sigma^{\prime}}$ and free ground state correlators

$$
\left\langle\Psi_{m \sigma}^{\dagger}(x) \Psi_{m^{\prime} \sigma^{\prime}}(0)\right\rangle=\left\langle\Psi_{m \sigma}(x) \Psi_{m^{\prime} \sigma^{\prime}}^{\dagger}(0)\right\rangle=\frac{\delta_{m m^{\prime}} \delta_{\sigma \sigma^{\prime}}}{2 \pi i x}
$$

Note that we follow PG in our choice of field normalization, which differs from that used by $\mathrm{AL}^{26}$ by $\Psi_{\text {here }}=$ $\psi_{\mathrm{AL}} / \sqrt{2 \pi}$. Consequently, our coupling constant is related to theirs by $\lambda_{\text {here }}=(2 \pi)^{2} \lambda_{\mathrm{AL}}$.

In the definition of $H_{\lambda}$, point splitting is needed because the product of two spin densities, $\vec{J}(x+\eta) \cdot \vec{J}(x)$, diverges with decreasing seperation $\eta$ between their arguments. To make this explicit, we use Wick's theorem,

$$
: A B:: C D:=: A B C D:+: A B C D:+: A B C D:+: A B C D:
$$

to rewrite the product of spin densities as follows:

$$
\begin{aligned}
\vec{J}(x+\eta) \cdot \vec{J}(x)= & \frac{1}{4} \sum_{m \sigma \sigma^{\prime}} \sum_{m^{\prime} \bar{\sigma} \bar{\sigma}^{\prime}}: \Psi_{m \sigma}^{\dagger}(x+\eta) \vec{\tau}_{\sigma \sigma^{\prime}} \Psi_{m \sigma^{\prime}}(x+\eta):: \Psi_{m^{\prime} \bar{\sigma}}^{\dagger}(x) \vec{\tau}_{\bar{\sigma} \bar{\sigma}^{\prime}} \Psi_{m^{\prime} \bar{\sigma}^{\prime}}(x): \\
= & \frac{1}{4} \sum_{m \sigma \sigma^{\prime}} \sum_{m^{\prime} \bar{\sigma} \bar{\sigma}^{\prime}} \vec{\tau}_{\sigma \sigma^{\prime}} \cdot \vec{\tau}_{\bar{\sigma} \bar{\sigma}^{\prime}}\left[: \Psi_{m \sigma}^{\dagger}(x+\eta) \Psi_{m \sigma^{\prime}}(x+\eta) \Psi_{m^{\prime} \bar{\sigma}}^{\dagger}(x) \Psi_{m^{\prime} \bar{\sigma}^{\prime}}(x):\right. \\
& \left.+\frac{\delta_{m m^{\prime}}}{2 \pi i \eta}\left(\delta_{\sigma^{\prime} \bar{\sigma}}: \Psi_{m \sigma}^{\dagger}(x+\eta) \Psi_{m \bar{\sigma}^{\prime}}(x):+\delta_{\sigma \bar{\sigma}^{\prime}}: \Psi_{m \sigma^{\prime}}(x+\eta) \Psi_{m \bar{\sigma}}^{\dagger}(x):\right)+\frac{\delta_{\sigma \bar{\sigma}^{\prime}} \delta_{\sigma^{\prime} \bar{\sigma}} \delta_{m m^{\prime}}}{(2 \pi i \eta)^{2}}\right]
\end{aligned}
$$

The point-splitting prescription in Eq. (A.1) subtracts off the $1 / \eta^{2}$ divergence of the last term of Eq. (A.6b). The contributions of the second and first terms to $H_{\lambda}$ can be organized as $H_{\lambda}=H_{1}+H_{\text {int }}$, describing single-particle 
elastic scattering and two-particle interactions, respectively. Taking $x=0$ and $\eta \rightarrow 0$, we find:

$$
\begin{aligned}
H_{1} & =-\frac{\lambda}{8 \pi i} \lim _{\eta \rightarrow 0} \sum_{m \sigma \sigma^{\prime}}: \frac{1}{\eta}\left[\Psi_{m \sigma}^{\dagger}(\eta) \vec{\tau}_{\sigma \sigma^{\prime}}^{2} \Psi_{m \sigma^{\prime}}(0)-\Psi_{m \sigma^{\prime}}^{\dagger}(0) \vec{\tau}_{\sigma^{\prime} \sigma}^{2} \Psi_{m \sigma}(\eta)\right]: \\
& =-\frac{3 \lambda}{8 \pi i} \lim _{\eta \rightarrow 0} \sum_{m \sigma}:\left[\frac{1}{\eta}\left(\Psi_{m \sigma}^{\dagger}(\eta)-\Psi_{m \sigma}^{\dagger}(0)\right) \Psi_{m \sigma}(0)-\Psi_{m \sigma}^{\dagger}(0) \frac{1}{\eta}\left(\Psi_{m \sigma}(\eta)-\Psi_{m \sigma}(0)\right)\right]: \\
& =-\frac{3 \lambda}{8 \pi i} \sum_{m \sigma}:\left[\left(\partial_{x} \Psi_{m \sigma}^{\dagger}\right)(0) \Psi_{m \sigma}(0)-\Psi_{m \sigma}^{\dagger}(0)\left(\partial_{x} \Psi_{m \sigma}\right)(0)\right]:
\end{aligned}
$$

$$
H_{\mathrm{int}}=-\lambda \sum_{m m^{\prime}}: \vec{J}_{m}(0) \cdot \vec{J}_{m^{\prime}}(0):
$$

To obtain Eq. (A.7b), we used $\vec{\tau}_{\sigma \sigma^{\prime}}^{2}=3 \delta_{\sigma \sigma^{\prime}}$ and subtracted and added $: \Psi_{m \sigma}^{\dagger}(0) \Psi_{m \sigma}(0)$ : inside the square brackets. Now pass to the momentum representation, using Eq. (A.4) and the shorthand notations (following $\mathrm{PG}^{29}$ )

$$
\begin{aligned}
\rho_{m \sigma}(0) & =\frac{1}{L} \rho_{m \sigma}, & \rho_{m \sigma} & =\sum_{k k^{\prime}} \psi_{k m \sigma}^{\dagger} \psi_{k^{\prime} m \sigma}, \\
\vec{J}_{m}(0) & =\frac{1}{L} \vec{j}_{m}, & \vec{j}_{m} & =\frac{1}{2} \sum_{k k^{\prime} \sigma \sigma^{\prime}} \psi_{k m \sigma}^{\dagger} \vec{\tau}_{\sigma \sigma^{\prime}} \psi_{k^{\prime} m \sigma^{\prime}},
\end{aligned}
$$

for the conduction electron channel- $m$ charge and spin densities at the impurity. This gives

$$
\begin{aligned}
H_{1} & =-\frac{\alpha_{1}}{2 \pi \nu} \sum_{m \sigma k k^{\prime}}\left(\xi_{k}+\xi_{k^{\prime}}\right): \psi_{k m \sigma}^{\dagger} \psi_{k^{\prime} m \sigma}: \\
H_{\mathrm{int}} & =-\frac{2 \phi_{1}}{3 \pi \nu^{2}} \sum_{m m^{\prime}}: \vec{j}_{m} \cdot \vec{j}_{m^{\prime}}:
\end{aligned}
$$

Here $\nu=L /\left(2 \pi \hbar v_{\mathrm{F}}\right)$ is the extensive 1D density of states per spin and channel, and the prefactors were expressed in terms of the constants

$$
\alpha_{1}=\phi_{1}=\frac{3 \lambda}{8 \pi\left(\hbar v_{\mathrm{F}}\right)^{2}}=\frac{1}{T_{\mathrm{K}}} .
$$

(This notation is consistent with that of Ref. 46, where $H_{\lambda}$ served starting point for calculating Fermi-liquid corrections, too.) Checking dimensions, with $\left[H_{\lambda}\right]=\mathcal{E}$ and $\left[\Psi_{m \sigma}\right]=1 / \sqrt{\mathcal{L}}(\mathcal{E}$ stands for energy, $\mathcal{L}$ for length $)$, we see that $[\lambda]=\mathcal{E} \mathcal{L}^{2}$. Since $[\nu]=1 / \mathcal{E},\left[\hbar v_{\mathrm{F}}\right]=\mathcal{E} \mathcal{L}$, we have $\left[\alpha_{1}\right]=\left[\phi_{1}\right]=1 / \mathcal{E}$, thus, $\alpha_{1}$ and $\phi_{1}$ have dimensions of inverse energy. In the main text, they are identified with $1 / T_{\mathrm{K}}$; in fact, the numerical prefactor in Eq. (A.11) is purposefully chosen such that the leading term in the expansion $(25)$ of the phase shift $\tilde{\delta}_{m \sigma}(\varepsilon)$ turns out to take the form $\varepsilon / T_{\mathrm{K}}$.

To elucidate how the case $N>1$ differs from $N=1$, we write $H_{\text {int }}=H_{2}+H_{3}$ in the main text, with $H_{2}$ and $H_{3}$ given in Eqs. (15b) and (15c), respectively, where $H_{3}$ occurs only for $N>1$.
1 W. J. de Haas, J. de Boer, and G. J. van den Berg, Physica 1, 1115 (1934).

2 W. J. de Haas and G. J. van den Berg, Physica 3, 440 (1936).

3 J. Kondo, Prog. Theor. Phys. 32, 37 (1964).

4 T. A. Costi, L. Bergqvist, A. Weichselbaum, J. von Delft, T. Micklitz, A. Rosch, P. Mavropoulos, P. H. Dederichs, F. Mallet, L. Saminadayar, and C. Bäuerle, Phys. Rev. Lett. 102, 056802 (2009).

5 M. Hanl, A. Weichselbaum, T. A. Costi, F. Mallet, L. Saminadayar, C. Bäuerle, and J. von Delft, Phys. Rev. B 88, 075146 (2013)

${ }^{6}$ F. Mallet, J. Ericsson, D. Mailly, S. Ünlübayir, D. Reuter, A. Melnikov, A. D. Wieck, T. Micklitz, A. Rosch, T. A. Costi, L. Saminadayar, and C. Bäuerle, Phys. Rev. Lett. 97, 226804 (2006).

7 K. G. Wilson, Rev. Mod. Phys. 47, 773 (1975).

${ }^{8}$ H. R. Krishna-murthy, J. W. Wilkins, and K. G. Wilson, Phys. Rev. B 211003 (1980).

9 A. Weichselbaum and J. von Delft, Phys. Rev. Lett. 99 076402 (2007).

10 R. Bulla, T. A. Costi, and T. Pruschke, Rev. Mod. Phys.
80, 395-450 (2008).

11 A. Weichselbaum, Ann. Phys. 327, 2972-3047 (2012).

12 C. P. Moca, A. Alex, J. von Delft, and G. Zaránd, Phys. Rev. B, 86, 195128 (2012).

13 Ph. Nozières, J. Low Temp. Phys. 17, 31 (1974).

$14 \mathrm{Ph}$. Nozières, in Proceedings of the 14 th International Conference on Low Temperature Physics, edited by M. Krusius and M. Vuorio, (North-Holland, Amsterdam, 1974) Vol. 5, p. 339.

$15 \mathrm{Ph}$. Nozières and A. Blandin, J. de Physique 41, 193 (1980).

16 D. Cragg and P. Lloyd, J. Phys. C, 11, L-597 (1978).

17 D. Cragg and P. Lloyd, J. Phys. C, 12, L-215 (1979).

18 D.M. Cragg, P. Lloyd, and P. Nozières, J. Phys. C, 13, 243 (1980).

19 I. Affleck, A. W. W. Ludwig, H.-B. Pang, and D.L. Cox, Phys. Rev. B, 45, 7918-7935 (1992).

20 Y. Nishikawa and A. C. Hewson, Phys. Rev. B B 86, 245131 (2012).

21 A. Yoshimori, Prog. Theor. Phys. 55, 67 (1976).

22 L. Mihaly and A. Zawadowski, J. de Physique 39, 483 (1978). 
23 A.M. Tsvelick and P.B. Wiegmann, Adv. Phys. 32, 45 (1983).

24 N. Andrei, K. Furuya, and J.H. Lowenstein, Rev. Mod. Phys. 55, 331 (1983).

25 I. Affleck, Nucl. Phys. B 336, 517 (1990)

${ }^{26}$ I. Affleck and A. W. W. Ludwig, Phys. Rev. B 48, 7297 (1993).

27 Y. Nishikawa, D. J. G. Crow, and A.C. Hewson, Phys. Rev. B 82, 115123 (2010).

28 M. Pustilnik and L. I. Glazman, Phys. Rev. Lett., 87, 216601 (2001).

29 M. Pustilnik and L.I. Glazman, J. Phys.: Cond. Mat. 16, R513 (2004).

30 L. Glazman and M. Pustilnik, in Nanophysics: Coherence and Transport, edited by H. Bouchiat et al. (Elsevier, Amsterdam, 2005), pp. 427-478.

31 C. Mora, Phys. Rev. B 80, 125304 (2009).

32 C. Mora, P. Vitushinsky, X. Leyronas, A. A. Clerk, and K. Le Hur, Phys. Rev. B 80, 155322 (2009).

33 A. K. Mitchell and E. Sela, Phys. Rev. B 85, 235127 (2012).

34 M.Hanl and A. Weichselbaum, Phys. Rev. B 89, 075130 (2014).

35 The Drude conductivity of a disordered metal has the form $\rho_{\text {tot }}^{-1} \propto \int d \varepsilon \tau(\varepsilon)\left(-\partial_{\varepsilon} f(\varepsilon, T)\right)$. In the presence of magnetic impurities (but neglecting phonons), the total scattering rate is the sum of the scattering rates due static disorder and magnetic impurities, $1 / \tau(\varepsilon)=1 / \tau_{\text {st }}+1 / \tau_{\text {mag }}(\varepsilon)$, with $1 / \tau_{\text {mag }}(\varepsilon) \propto \sum_{m \sigma} A_{m \sigma}(\varepsilon)$. Eq. (2) is obtained ${ }^{36}$ by expanding $\tau(\epsilon)$ in powers of the ratio $\tau_{\text {st }} / \tau_{\text {mag }}(\varepsilon) \ll 1$, and correspondingly expanding the resisitity as $\rho_{\text {tot }}=$ $\rho_{\text {st }}+\rho(T, B)$, where the second contribution, due to magnetic impurities, is much smaller than the first.

36 A.A. Abrikosov, Physis, 2, 5 (1965).

37 T. A. Costi, Phys. Rev. Lett. 85, 1504-1507, (2000).

38 A. Rosch, T. A. Costi, J. Paaske, and P. Wölfle, Phys. Rev. B 68, 014430, (2003).

39 A.V. Kretinin, H. Shtrikman, D.A. Goldhaber-Gordon, M. Hanl, A. Weichselbaum, J. von Delft, T.A. Costi, and D. Mahalu, Phys. Rev. B, 84, 245316 (2011).

40 F. B. Anders and A. Schiller, Phys. Rev. Lett. 95, 196801 (2005).

41 R. Peters, T. Pruschke, and F. B. Anders, Phys. Rev. B 74, 245114 (2006).

42 A. Weichselbaum, Phys. Rev. B 86, 245124 (2012).

43 A. Weichselbaum, Phys. Rev. B 84, 125130 (2011).

44 L. N. Oliveira, V. L. Libero, H. O. Frota and M. Yoshida, Physica B 171, 61 (1991).

${ }^{45}$ F. Bauer, J. Heyder, E. Schubert, D. Borowsky, D. Taubert, B. Bruognolo, D. Schuh, W. Wegscheider, J.von Delft, and S. Ludwig, Nature (London), 501, 73, (2013).

${ }^{46}$ C. B. M. Hörig, C. Mora, and D. Schuricht, Phys. Rev. B 89, $165411(2014)$

47 I. Affleck, Nucl. Phys. B, 265, 409 (1986).

48 A. W. W. Ludwig, Methods of Conformal Field Theory in Condensed Matter Physics An Introduction to NonAbelian Bosonization, Lecture Notes, Trieste 1992 (unpublished)

49 Jan von Delft, PhD thesis, Cornell University, 1995. 